\title{
Advances in Femtosecond Optical Spectroscopy Techniques
}

\author{
C. V. SHANK \\ Bell Telephone Laboratories, Holmdel, New Jersey 07733
}

This paper reviews the recent advances in optical pulse measurement techniques which have made possible the investigation of ultrafast phenomena in condensed matter on a time scale of 100 femtoseconds or less. Recent results in pulse generation, amplification, white light continuum generation, and pulse compression will be discussed. In addition, applications of these pulses for measurement will be discussed.

\section{INTRODUCTION}

Recent advances in optical pulse generation and measurement techniques have opened up the possibility of making time resolved measurements in photochemistry, biology and physics with a time resolution of well under 100 femtoseconds. In this paper I will describe optical pulse generation techniques which have led to the generation of optical pulses as short as 30 femto seconds. ${ }^{1}$ Also, the application of these optical pulses to the measurement of ultrafast processes will be described.

In the last decade and a half rapid progress has been made in optical pulse generation methods. In the late 1960 s the first optical pulses in the picosecond range ${ }^{2}$ were produced and applied to investigate transient processes in matter. With the advent of the passively modelocked $\mathrm{cw}$ dye laser in the mid 1970 s subpicosecond ${ }^{3}$ measurements became a reality. Now in the last year and a half new technologies have been devised which permit investigations with a time resolution of less than 100 femtoseconds. With the advent of the colliding pulse dye laser ${ }^{4}$ we can now directly generate optical pulses 
on the order of 60 femto seconds and with amplification ${ }^{5}$ techniques produce pulses with an intensity of several gigawatts. Applying optical compression techniques we have been able to shrink the pulse width down to 30 femtoseconds.

\section{OPTICAL PULSE TECHNOLOGY}

A major step in the progress of pulse generation with dye lasers has been the development of the colliding pulse dye laser. In Figure 1 we show the optical configuration for a passively modelocked dye laser in the colliding pulse configuration. The laser cavity is in a ring configuration containing two elements: an optically pumped saturable gain medium and a saturable absorber dye. The amazing simplicity of this laser, in part, accounts for its ability to generate femtosecond optical pulses. By minimizing the amount of material in the cavity the effects of group velocity dispersion are reduced allowing the cavity to sustain a broad bandwidth of oscillating frequencies necessary to form a short optical pulse. The mechanism ${ }^{6}$ for pulse shortening in the colliding pulse laser is similar to that for the passively modelocked dye laser. Optical pulse shaping takes place by preferential absorption of the leading edge of the pulse by the saturable absorber and preferential amplification of the leading edge of the optical pulse by

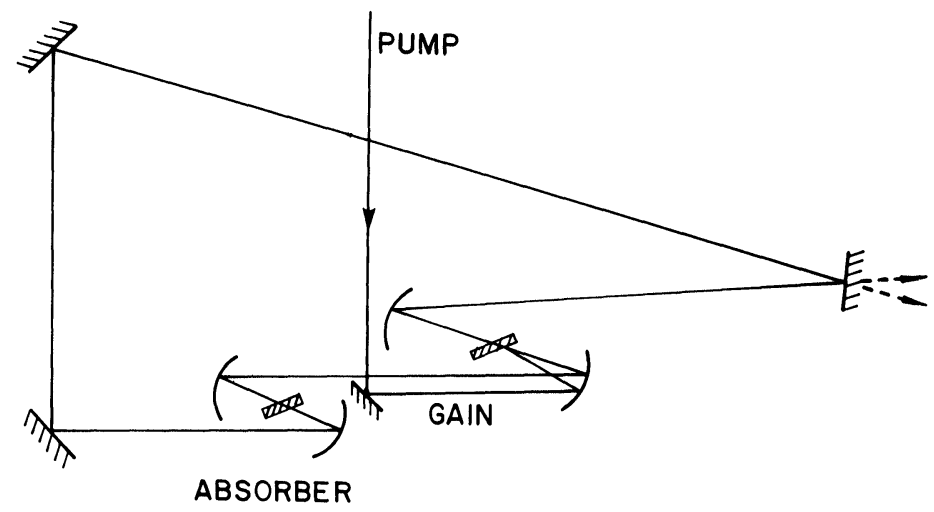

FIGURE 1 Diagram of the optical configuration of a colliding pulse modelocked laser. The pump is a continuous Argon laser. 
the gain dye, which combined with cavity loss, effectively shortens the pulse with each pass around the cavity until the limiting pulsewidth is achieved. The additional mechanism operative in the colliding pulse configuration is the interaction of the two oppositely directed pulse streams present in the ring laser cavity. The two pulse streams "collide" in a thin 10 micron ethylene glycol stream containing the saturable absorber 3,3'-diethyloxydicarboxyanine iodide. Synchronization of the two counterpropagating pulse streams occurs because the minimum energy is lost in the saturable absorber when the pulses meet in the absorber. The pulses interfere and set up a standing wave pattern in the absorber stream. The standing wave field minimizes the energy lost because the field is most intense where the absorption is saturated, whereas the electric field is weakest in the unsaturated field minima. The shortest optical pulses are produced with a thin saturable absorber which confines the standing wave field. In Figure 2 we have plotted the experimentally measured autocorrelation function using second harmonic generation in a crystal of KDP. The optical pulsewidth determined from the autocorrelation measurement is 65 femtoseconds.

Even shorter optical pulses have been generated using pulse compression techniques. More than a decade ago Gires and Tournois ${ }^{7}$ and Giordmaine et $a l^{8}{ }^{8}$ proposed shortening of optical pulses using

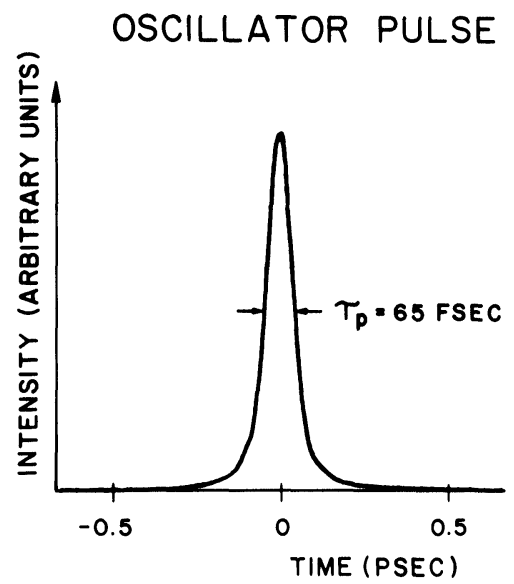

FIGURE 2 The experimentally measured autocorrelation function using the second harmonic upconversion technique. 
compression techniques analogous to those used at microwave frequencies. Optical pulse compression is achieved by passing the pulse to be compressed through an optical Kerr medium followed by a dispersive delay line. When an intense optical pulse is passed through the nonlinear medium the refractive index is modified by the electric field $E$.

$$
n=n_{0}+n_{2}\left\langle E^{2}\right\rangle
$$

A phase change is impressed on the pulse given by

$$
\delta \phi=n_{2}\left\langle E^{2}\right\rangle \omega z / c
$$

where $\omega$ is the frequency and $z$ is the distance traveled in the Kerr medium. Since the pulse intensity rises very steeply a frequency sweep or "chirp" is impressed on the pulse carrier frequency given by

$$
\delta \omega=\left(\omega z n_{2} / c\right) d / d t\left\langle E^{2}(t)\right\rangle
$$

One problem with this scheme is that other nonlinear processes such as self focusing can interfere with the pulse compression process. These problems can be overcome by using an optical fiber., ${ }^{9,10,11}$

To reassemble the chirped optical pulse a dispersive delay line is required. A nearly total linearly dispersive delay line is a parallel pair of gratings. Each wavelength passing through the pair is diffracted at a different angle by the first grating of the pair and recollimated by the second grating. The end result is a wavelength dependent optical path delay.

In Figure 3 we have schematically drawn the optical set up for femtosecond pulse compression. Optical pulses of 90 femotoseconds in duration were focused into a $15 \mathrm{~cm}$ long polarization preserving single mode optical fiber. When a few microjoules of pulse energy

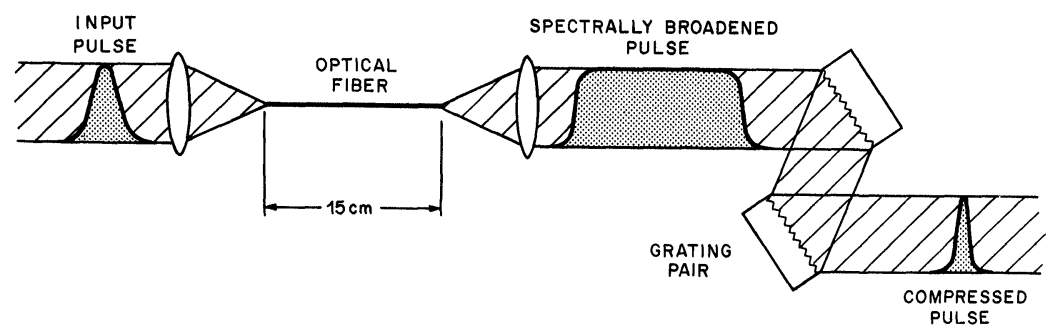

FIGURE 3 Schematic diagram pulse compression in an optical fiber. 
were coupled into the fiber, the frequency spectrum broadened significantly. At about $0.5 \mathrm{~nJ}$ energy coupled into the fiber the frequency spectrum was broadened by about a factor of three. The spectral halfwidth broadened from 60 Angstroms to 200 Angstroms. Optical compression was achieved by the grating pair. The grating pair also served the useful purpose of compensating the group dispersion in the pulse measurement optics. In Figure 4 we have plotted the measured autocorrelation function of the 30 femotosecond compressed optical pulse.

For many experiments it is desirable to amplify short optical pulses and to use nonlinear optical techniques to generate pulses at new frequencies. The peak pulse power coming directly from the dye laser oscillator is in the kilowatt range. An amplifier design that can amplify optical pulse to gigawatt powers and yet preserve the femtosecond pulsewidth is shown in Figure 5. A frequency doubled Nd:YAG laser at $530 \mathrm{~nm}$ is synchronized to a mode-locked dye laser, and used to pump a four stage dye amplifier. A saturable absorber dye isolates each stage. A grating pair is used to compensate the dispersion in the dye amplifiers and optical components. In Figure 6 we show the autocorrelation function of an amplified pulse having a gigawatt peak power and a pulsewidth of 70 femtoseconds.

A useful technique for generating ultrashort optical pulses at different frequencies is the process of continuum generation. A

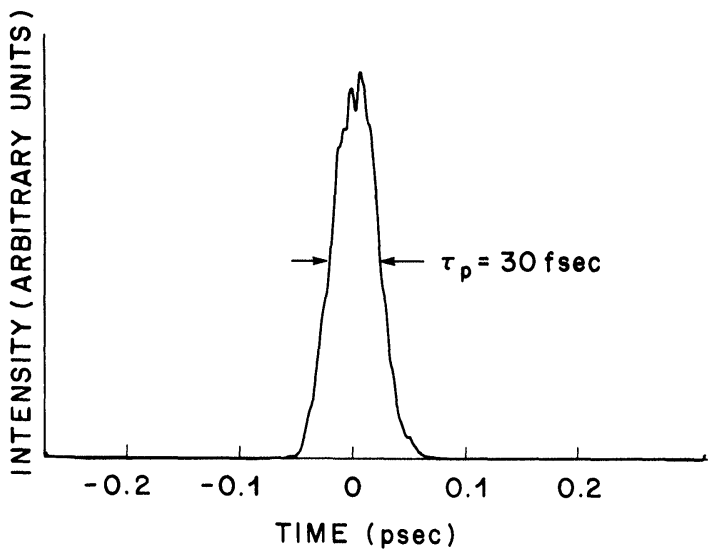

FIGURE 4 Autocorrelation function of a compressed 30 femtosecond optical pulse. 


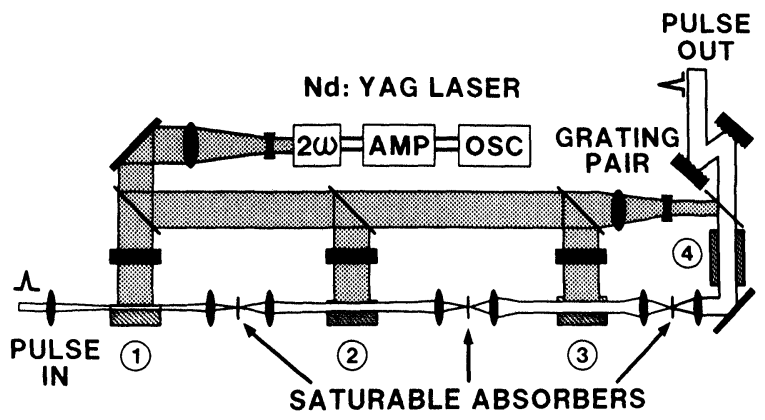

FIGURE 5 Layout for a four stage femtosecond pulse amplifier.

broad-band frequency continuum can be generated simply by focusing an intense short optical pulse into almost any transparent material. The precise mechanism for this generation process is still the subject of intense controversy.

One of the most important considerations for extending the white light continuum generation technique into the femtosecond time regime is a sweep in the time of the various frequency components brought about by group velocity dispersion. Some time sweep is inevitable because of the broad frequency range over which the

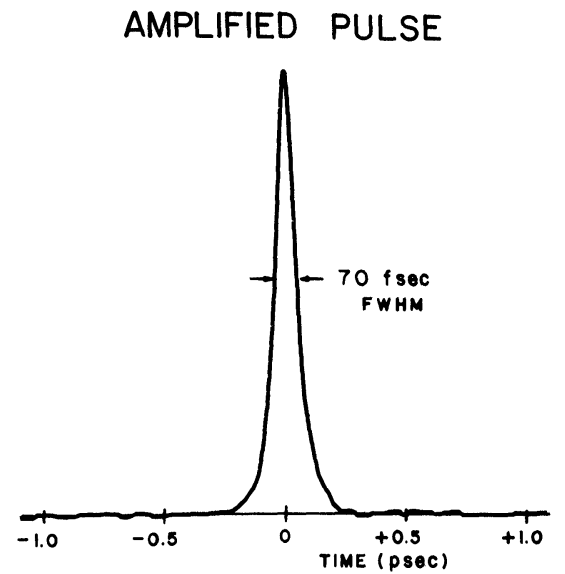

FIGURE 6 Autocorrelation function of an amplified pulse. 
continuum extends. This sweep can be greatly reduced by generating the continuum in the shortest possible path length and by eliminating lenses by using reflective optics. We have succeeded in generating a short, 80 femtosecond white light pulse by focusing an intense gigawatt 70 femtosecond optical pulse into a thin 400 micro thick free flowing stream of ethylene glycol. ${ }^{12}$ With an excitation wavelength of $620 \mathrm{~nm}$ the generated continuum pulse extends from 1900 Angstroms to 1.6 microns.

We have determined the frequency sweep in the continuum to be less than or comparable to the generated pulsewidth. Most of the observed frequency sweep is a result of the generation process itself. We observe the leading edge of the continuum to contain the red shifted portions of the continuum and the blue frequency to occur on the trailing edge of the generating pulse. This result suggests that the self phase modulation process we described in the section on pulse compression may play an important role in generating the white light pulses as well. The spectral width of the white light pulse is too large to be completely described by self phase modulation. At the intensities used to generate the continuum pulse $\left(10^{11}\right.$ watts $\left./ \mathrm{cm}^{2}\right)$, it is not too surprising that other nonlinear effects would contribute to the generation process.

\section{FEMTOSECOND SPECTROSCOPY}

An experimental arrangement for measuring time resolved spectra with femtosecond optical pulses is shown in Figure 7. An optical pulse having a wavelength of $620 \mathrm{~nm}$ is generated using a colliding pulse modelocked dye laser. These pulses are then amplified to gigawatt levels using the four stage amplifier described previously. The amplified pulses are divided into two parts, one for exciting and the other for probing the sample. The excitation pulses are passed through a nonlinear frequency shifting medium to generate the desired frequency. The frequency shifting can be achieved by using the stimulated Raman effect or some other nonlinear process. The probing pulses pass through a variable path or time delay controlled by a stepper motor. The pulses are then focused into an ethylene glycol stream to generate a white light continuum pulse. The white light continuum pulse is further divided in order to provide a measuring 


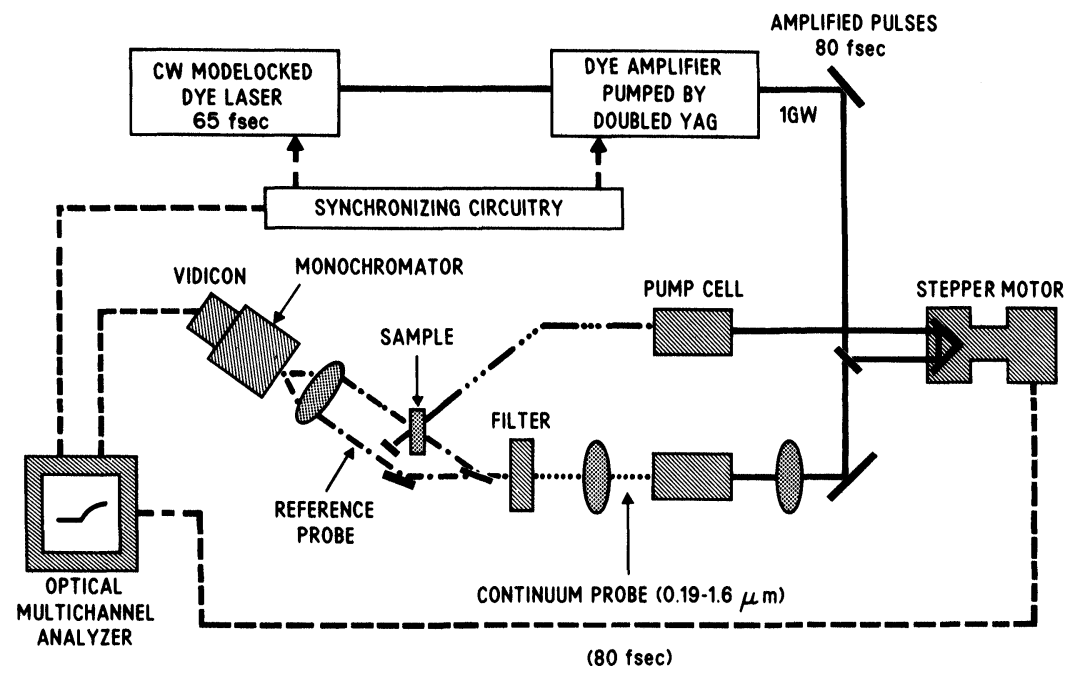

FIGURE 7 Experimental arrangement for time resolved spectroscopic measurements.

pulse and a reference pulse. The reference and measurement pulse are then directed into a spectrometer with a vidicon or optical multichannel analyzer on the output of the spectrometer. In this way, measurements of optically induced changes in absorption spectrum can be performed over a broad spectral range at different time delays following excitation as determined by the stepper motor controlled path delay. The resolution is determined by the convolution of the pumping and probing pulsewidths. Care must be taken to limit artifacts caused by group delay in the various parts of the white light continuum spectrum.

The application of the techniques just described cover a broad range of fields including chemistry, photobiology and physics. I will describe one recent application of femtosecond measurement techniques to a problem that is currently of interest to both physicists and chemists.

The molecule polyacetylene has been the subject of considerable interest because it is a prototype one dimensional semiconductor. ${ }^{13}$ A great volume of work has been reported in the literature on its optical and electronic properties, a detailed discussion of which is beyond the scope of this present article. 
Recently there has been extensive discussion concerning the nature of optically induced absorption ${ }^{14}$ in polyacetylene. Theoretical studies of $1 \mathrm{D}$ models of polyacetylene indicate that the electronic excitations are associated with localized large amplitude distortions of the ground state configuration of the long chain molecule. Changes in the optical absorption spectrum due to the creation of these distortions are characterized by the appearance of new absorption processes below the band gap, $E_{g}$ and a uniform reduction of the absorption above $E_{9}$. Although these models are highly idealized, their predictions are qualitatively similar to experimental observation. In Figure 8 we show a plot of the experimentally measured induced optical absorption spectrum following excitation of a thin film of trans isomer polyacetylene with a 90 femtosecond optical pulse with a wavelength of $620 \mathrm{~nm}$. Note that the frequency spectrum observed at 0.5 picoseconds is broader than the normalized spectrum taken at 200 picoseconds. Similar narrowing of absorption with time delay spectra has been observed by Greene et al. ${ }^{15}$ for induced absorption spectra in large molecules and has been attributed to vibrational relaxation.

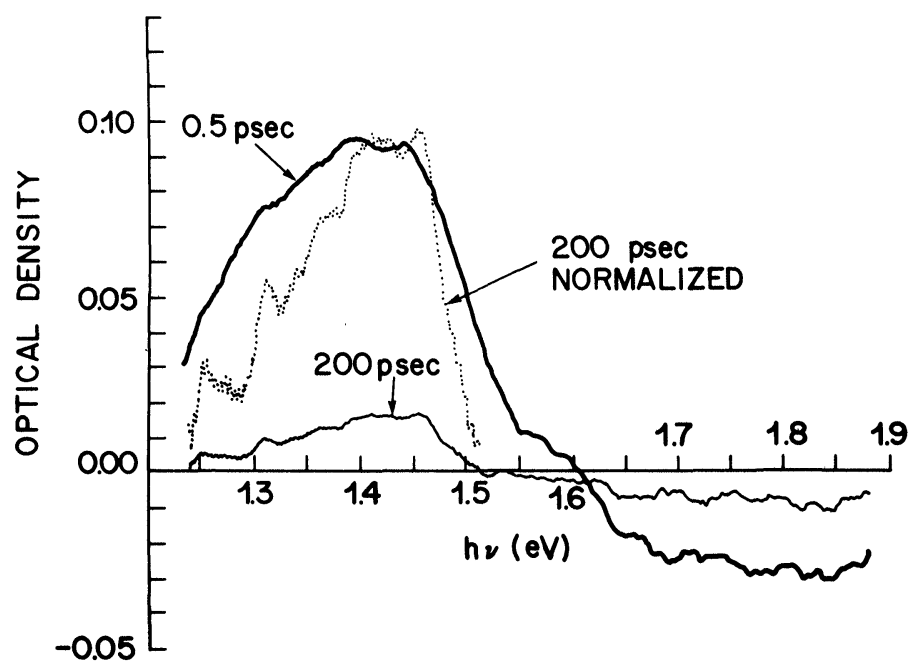

FIGURE 8 Induced optical absorption spectra of optically excited trans polyacetylene. 
In Figure 9 we have plotted the magnitude of the induced absorption measured at the peak as a function of time. The expanded plot shows the formation time for induced absorption is less than 150 femtoseconds. The decay of the absorption is nonexponential but closely fits a $1 / \sqrt{t}$ time dependence.

The observation that these absorption features are formed so rapidly (less than 150 femtoseconds) strongly suggests they arise from intrinsic excitations of the polyacetylene chain. The $1 / \sqrt{t}$ time dependence is what we would expect from a diffusive type recombination process.

We have considered a simple model in which photoexcited kink pairs execute one dimensional randon walks along the carbon chain before undergoing geminate recombination. The process can be described by two parameters, the jump rate, $\tau_{d}^{-1}$, and the ratio of the jump distance to the $1 \mathrm{D}$ recombination length. For simplicity we assume this ratio to be unity so that if the kinks approach within one jump distance they will recombine. Using this model we can describe the decay of the number of absorbing species by the expression

$$
N(t) N_{d}(t)=\operatorname{erf}\left(\sqrt{\frac{\tau d}{t}} / t\right)
$$

The result of this calculation is consistent with the $1 / \sqrt{t}$ time dependence seen in the data. At $300 \mathrm{~K}$ an experimental fit to the data yields $\tau_{d}=0.1$ picoseconds.

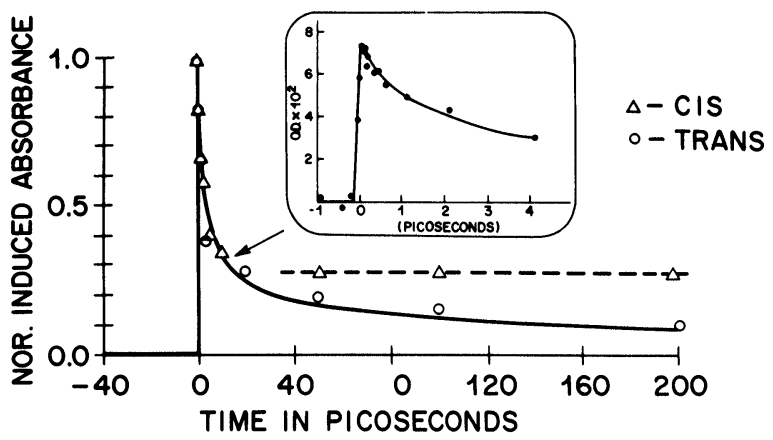

FIGURE 9 Time dependence of the peak induced absorbance of trans and cis isomers of polyacetylene. 


\section{CONCLUSION}

In this paper we have described the progress that has taken place in the development of measurement techniques for investigating ultrafast processes. These methods now give us the ability to make measurements with a resolution time of less than 100 femtoseconds. Optical pulse techniques are already being applied to important problems in a broad variety of fields. Only the future will tell whether or what important new discoveries await in the world of fast events that can be probed in femtosecond time regime.

\section{References}

1. C. V. Shank, R. L. Fork, R. Yen, R. H. Stolen and W. J. Tomlinson, Appl. Phys. Lett. 40, 9 (1982).

2. A. J. DeMaria, D. A. Stetser and H. Heynau, Appl. Phys. Lett. 8, 22 (1966).

3. C. V. Shank and E. P. Ippen, Appl. Phys. Lett. 24, 373 (1974).

4. R. L. Fork, B. I. Greene and C. V. Shank, Appl. Phys. Lett. 38, 671 (1981).

5. R. L. Fork, C. V. Shank and R. T. Yen, Appl. Phys. Lett. 41, 223 (1982).

6. G. H. C. New, IEEE Quantum Electron. QE-10, 115 (1974).

7. F. Gires and P. Tournois, Comp. Rend. 258, 6112 (1064).

8. J. A. Giordmaine, M. A. Duguay and J. W. Hansen, IEEE Quantum Electron. 4, 252 (1968).

9. R. H. Stolen and C. Lin, Phys. Rev. A 17,1448 (1978).

10. L. F. Mollenauer, R. H. Stolen and J. P. Gordon, Phys. Rev. Lett. 45, 1095 (1980).

11. H. Nakatsuka, D. Grischkowsky and A. C. Balant, Phys. Rev. Lett. 47, 1910 (1969).

12. R. L. Fork, C. V. Shank, C. Hirlimann, R. T. Yen and W. J. Tomlinson, to be published.

13. J. A. Heeger, Comments Solid State Phys. 10, 53 (1981).

14. J. Orenstein and G. L. Baker, Phys. Rev. Lett. 49, 1043 (1982).

15. B. I. Greene, R. M. Hochstrasser and R. B. Weisemann, J. Chem. Phys. 70, 1247 (1979). 